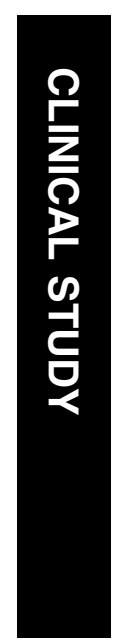

\title{
Pars plana vitrectomy for diabetic macular oedema: OCT and functional correlations
}

\begin{abstract}
Purpose A prospective study to evaluate the macular structural and functional effects of pars plana vitrectomy (PPV) for persistent diffuse clinically significant macular oedema (CSMO).
\end{abstract}

Method A total of 12 patients with persistent diffuse CSMO were recruited and underwent assessment including best-corrected visual acuity, fundus fluorescein angiography, optical coherence tomography (OCT) and fine matrix mapping (FMM) at baseline and over a period of a year poststandard three-port PPV.

Results The median baseline ETDRS letters score for all 12 patients was 52 (range 41-63) while at 12 months it had increased to 65 (range of 27-68), an improvement of two complete ETDRS lines $(P=0.037)$. Similarly, there was an improvement in the perifoveal cone thresholds $(P=0.02)$. The foveal thickening for all 12 patients ranged from a median of 183 to $751 \mu \mathrm{m}$ (normal range 126-

${ }^{1}$ Moorfields Eye Hospital, London, UK

${ }^{2}$ Department of Pathology, Institute of Ophthalmology, London, UK

Correspondence: Jl Patel, Department of Pathology, Institute of Ophthalmology, 11-43 Bath St., EC1V 9EL London, UK

Tel: +44207608 6808;

Fax: + 442076086862 .

E-mail: jigs37@

hotmail.com

Received: 23 August 2004 Accepted: 15 April 2005

Published online:

21 October 2005
$180 \mu \mathrm{m}$ ) and the macular volume ranged from a median of 2.13 to $6.42 \mathrm{~mm}^{3}$ (normal $<1.66 \mathrm{~mm}^{3}$ ). After surgery, both the median foveal thickness (from 334 to $280 \mu \mathrm{m}$ ) and median macular volume (from 3.24 to $2.61 \mathrm{~mm}^{3}$ ) demonstrated decreases over 12 months $(P=0.01)$. On baseline OCT, the patients fell into two anatomically distinct groups: Group $1(n=4)$ had a dome-shaped thickened macula with a partial posterior hyaloid separation and a significantly higher foveal thickness and macular volume than Group $2(n=8)$ which had a diffuse lowelevation profile of the thickened macula $(P=0.007)$.

Conclusions In this prospective study of PPV for persistent fovea-involving CSMO there was structural and functional improvement.
Il Patel ${ }^{1,2}$, PG Hykin'1, M Schadt', V Luong ${ }^{2}$, F Fitzke ${ }^{2}$ and ZJ Gregor ${ }^{1}$
Eye (2006) 20, 674-680. doi:10.1038/sj.eye.6701945; published online 21 October 2005

Keywords: diabetes; macular oedema; pars plana vitrectomy; OCT

\section{Introduction}

Diabetes mellitus is the leading cause of blindness in the working population in the developed world and diabetic macular oedema (DMO) accounts for $72 \%$ of registrable blindness among patients with diabetes mellitus. ${ }^{1}$ In all $9 \%$ of the diabetic population develop oedema within one disc diameter (DD) of the fovea and $40 \%$ of those have central macular involvement. ${ }^{2}$ The natural history of $\mathrm{DMO}$ is one of progressive visual loss with more than $50 \%$ of patients losing two or more lines of visual acuity within 2 years. ${ }^{3}$

At present there are only two proven modalities of management: strict control of systemic blood glucose and blood pressure has been shown to delay the onset and to reduce the progression of diabetic retinopathy for both Type I and Type II diabetes mellitus. ${ }^{4,5}$ The second is the timely use of photocoagulation of the perifoveal retina. ${ }^{6}$ The results of the Early Treatment Diabetic Retinopathy Study (ETDRS) showed that laser treatment mostly stabilised vision and reduced the risk of moderate visual loss by $50 \%$. However, only $3 \%$ of the patients improved by three or more lines of vision by the end of that study.

Lewis was the first to describe a positive effect of pars plana vitrectomy (PPV) on diabetic patients who had a thickened and taut posterior hyaloid membrane with traction on the macula. There were improvements in both vision and resolution of macular oedema ${ }^{7}$ and the 
accompanying shallow macular detachment thought to develop secondary to the tractional forces. ${ }^{7}$ Recently, reports of PPV performed on patients with an attached but non-thickened posterior hyaloid and without ophthalmoscopic evidence of macular traction showed improvement in the visual acuity and reduction of macular oedema. ${ }^{8,9}$ However, most of the studies on patients with a nonthickened posterior hyaloid membrane and DMO were retrospective and variable in surgical technique, with some patients having cataract ${ }^{9}$ or internal limiting membrane ${ }^{10}$ removed simultaneously.

The aim of this prospective study was to determine the clinical efficacy of PPV for patients with persistent and diffuse DMO in the presence of an attached but nonthickened posterior vitreous cortex.

\section{Patients and methods}

Patients with persistent clinically significant macular oedema (CSMO) despite previous macular laser, were offered enrolment in this prospective study. A masked observer assessed the best-corrected visual acuity (BCVA) for both Snellen and ETDRS vision score. All patients underwent dilated retinal examination, intravenous FFA, optical coherence tomography (using OCT 2) and fine matrix mapping (FMM). The preoperative examinations were performed within a month of surgery to obtain the baseline clinical parameters and then subsequently at 2, 6, 12 and 24, and 48 weeks postoperatively.

Inclusion criteria were (i) persistent diffuse CSMO involving the foveal centre of less than 2 years in duration (ii) previous treatment with macular laser and (iii) ETDRS vision score of 59 to 35 (equivalent Snellen visual acuity $6 / 15$ to $6 / 60$ ). Exclusion criteria were (i) posterior vitreous detachment diagnosed by the presence of the Weiss ring (ii) macular traction as evidenced by retinal striae involving the foveal centre (iii) macular ischaemia as defined by an enlarged foveolar avascular zone (FAZ $>1000 \mu \mathrm{m}$ ) or significant perifoveal capillary loss on fundus fluorescein angiography (FFA) and (iv) coexistent retinal disease.

OCT is a noninvasive, noncontact imaging modality producing high-resolution cross-sectional tomographs of ocular tissue. ${ }^{11}$ It produces a two-dimensional false colour image of the back-scattered light from different layers of the retina analogous to ultrasonic B-scan imaging. The only difference is that optical reflectivity is measured. Axial resolution up to $14 \mu \mathrm{m}$ is achievable. Six $6 \mathrm{~mm}$ diameter radial scans centred on the point of fixation were taken at each time point, assessed qualitatively, and analysed quantitatively using the retinal mapping software. Quantitative assessment using the computer software (Zeiss-Humphrey, Dublin, CA, software version A5, using model OCT 2) included an estimation of the foveal thickness and the macular volume, which is derived by an integration process of the average retinal thickness of the six radial scans.

FMM is a psychophysical method of examination, which determines the cone thresholds in a $9 \times 9$ degree field centred on the point of fixation. A total of 100 points within this test field are analysed using a modified Humphrey field analyser. A three-dimensional representation of photopic white stimulus thresholds (given as log units) can be generated from there data. The thresholds are a mean of the 100 points. The lower the threshold, the better the overall cone function within the tested field. This psychophysical analysis of photoreceptor function has been used and validated in the assessment macular function in central serous retinopathy ${ }^{12}$ and age-related macular degeneration. ${ }^{13}$ Overlay of the central OCT field on the central $9 \times 9$ degree field of the fine matrix map (after appropriate scaling and adjustment) was performed to evaluate corresponding retinal thickness and the associated cone function, and the changes in these parameters after PPV.

All patients underwent standard three-port PPV with elevation and the removal of the posterior vitreous cortex, but with no peeling of the internal limiting membrane. Fluid- $\mathrm{SF}_{6}$ gas exchange was performed if retinal breaks were found on the $360^{\circ}$ examination of the peripheral retina prior to the conclusion of the operation. Such breaks were treated with laser photocoagulation or cryotherapy. Subconjunctival injection of Betamethasone and Cefuroxime was given at the conclusion of the operation. Patients were treated with topical Atropine $1 \%$, Dexamethasone and Chloramphenicol for 3 weeks after the operation.

All patients were recruited in accordance with the Declaration of Helsinki and with the approval of the Ethics Committee of Moorfields Eye Hospital.

\section{Statistical analysis}

OCT and clinical parameters were analysed by unpaired Mann-Whitney $U$-test and a paired Wilcoxon test used for the appropriate comparison with the significance value taken at $P<0.05$.

\section{Results}

Seven male and five female patients were enrolled in the study. The average age of the patient was 63 years (range 46-72 years). All patients had type II diabetes mellitus of an average duration of 11 years (range 4-23 years). Four were on oral medication; six were on insulin, only one was on combined insulin and oral medication treatment, 
and one was controlled by diet alone. The average HbA1c was 8\% (range 5.8-9.9). Nine patients had controlled systemic hypertension and one was on lipid-lowering drugs. The patients suffered from no other significant ocular conditions. All patients had moderate nonproliferative diabetic retinopathy. All had fovea-involving macular oedema, which had been present for an average of 15 months (range 12-18 months) and all were previously treated with macular argon laser photocoagulation, on average three times (range 1-5) prior to surgery.

Per operative assessment confirmed an attached posterior hyaloid face in all cases and posterior hyaloid separation was induced as part of the operative protocol. There were no complications resulting from the surgery.

\section{OCT findings}

Whole group

The foveal thickening for all 12 patients ranged from 183 to $751 \mu \mathrm{m}$ (normal range $126-180 \mu \mathrm{m}$ ) and the macular volume ranged from 2.13 to $6.42 \mathrm{~mm}^{3}$ (normal $<1.66 \mathrm{~mm}^{3}$ ) (normal macular structural ranges ${ }^{14,15}$ ).

After surgery, for all 12 patients, both the median foveal thickness ( $334 \mu \mathrm{m}$ at baseline and $280 \mu \mathrm{m}$ at 12 months) and the median macular volume (3.24 at baseline and $2.61 \mathrm{~mm}^{3}$ at 12 months) showed gradual improvement over the 12-month postoperative period (the difference between baseline and 12 month levels for both parameters was significant, $P=0.01$ ) (Figure 1).

\section{Sub-group OCT analysis}

There appeared to be two distinct patterns of macular profile on OCT and the patients were divided into two groups accordingly. Group $1(n=4)$ had a dome-shaped thickened macula with posterior hyaloid partially elevated but adherent to the tip of the fovea and with a large central pocket of fluid within the retina (see Figure 2). Baseline median foveal thickness was $478 \mu \mathrm{m}$ (range $472-751 \mu \mathrm{m}$ ) and macular volume was $3.9 \mathrm{~mm}^{3}$ (range $\left.3.74-6.42 \mathrm{~mm}^{3}\right)$. Group $2(n=8)$ had a diffuse low-elevation profile of the thickened macula with reduced intraretinal reflectivity and multiple intraretinal cysts and no signs of a focal posterior hyaloid separation (see Figure 2). Baseline median foveal thickness was $233 \mu \mathrm{m}$ (range 198-374 $\mu \mathrm{m}$ ) and macular volume was $2.7 \mathrm{~mm}^{3}$ (range $2.13-3.52 \mathrm{~mm}^{3}$ ). The difference in the pre operative foveal thickness and macular volumes between the two groups was statistically significant $(P=0.007)$.

\section{Sub-group postoperative analysis}

In Group 1, the median foveal thickness showed an overall gradual decrease over the 12 months, from a baseline median of 478 to a median of $348 \mu \mathrm{m}$ (see Figure 3). For Group 2, there was little change in the foveal thickness throughout the postoperative period (from a median of 233 to a median of $213 \mu \mathrm{m}$ ) (see Figure 3). The difference in foveal thickness between the two groups at 12 months postoperatively was significant $(P=0.003)$.

The macular volume changes for Group 1 and Group 2 reflected the foveal thickness changes post surgery: a gradual decrease over 12 months for Group 1; little change for Group 2 (Figure 3). Unlike for foveal thickness, the difference in the 12-month postoperative volumes between the two groups was not significant $(P=0.06)$.

\section{Visual acuity results}

\section{Whole group}

The median baseline ETDRS letters score for all 12 patients was 52 (range 41-63) or a median Snellen acuity of $6 / 30$ (range $6 / 15$ to $6 / 60$ ). At 12 months, the ETDRS letters read increased to 65 (range of 27-68), an improvement of 13 letters or two complete ETDRS
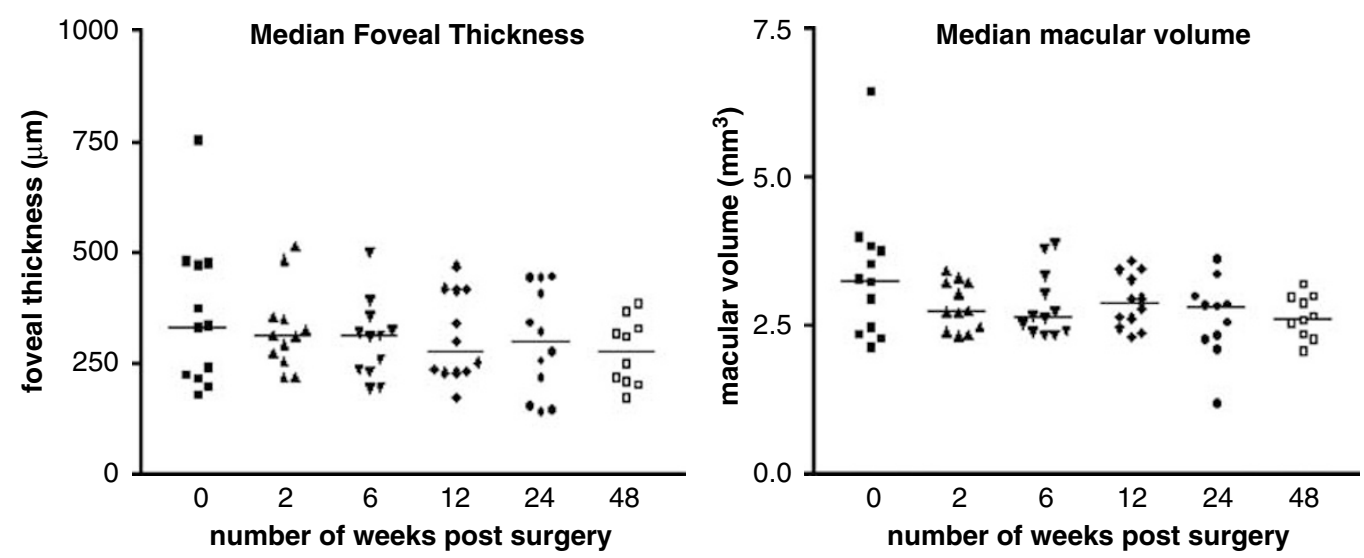

Figure 1 The median macular structural changes postvitrectomy in all 12 patients showing gradual improvement over the 1-year follow-up period. 
Group 1

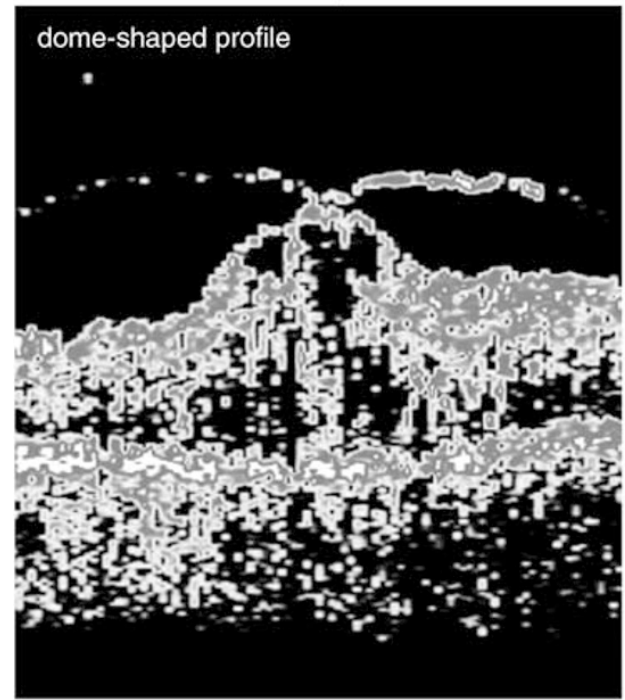

Group 2

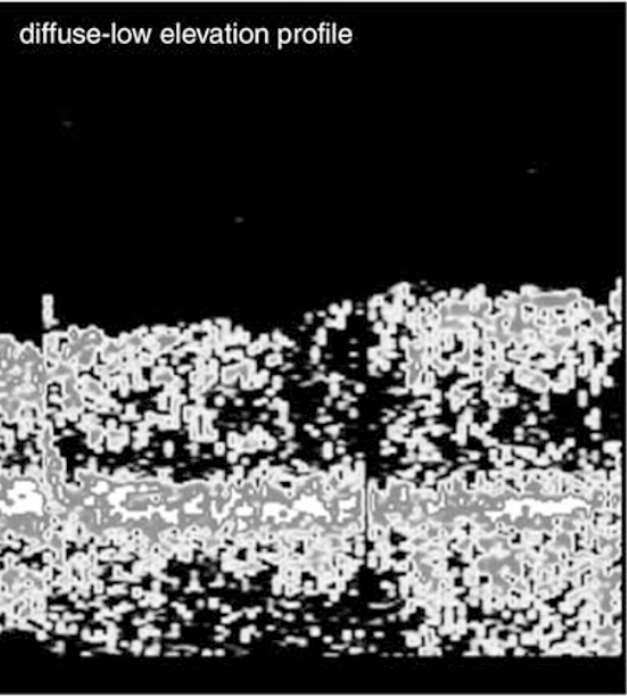

Figure 2 The two OCT defined macular profiles. Group 1 with its dome-shaped profile and Group 2, the diffuse low elevation.
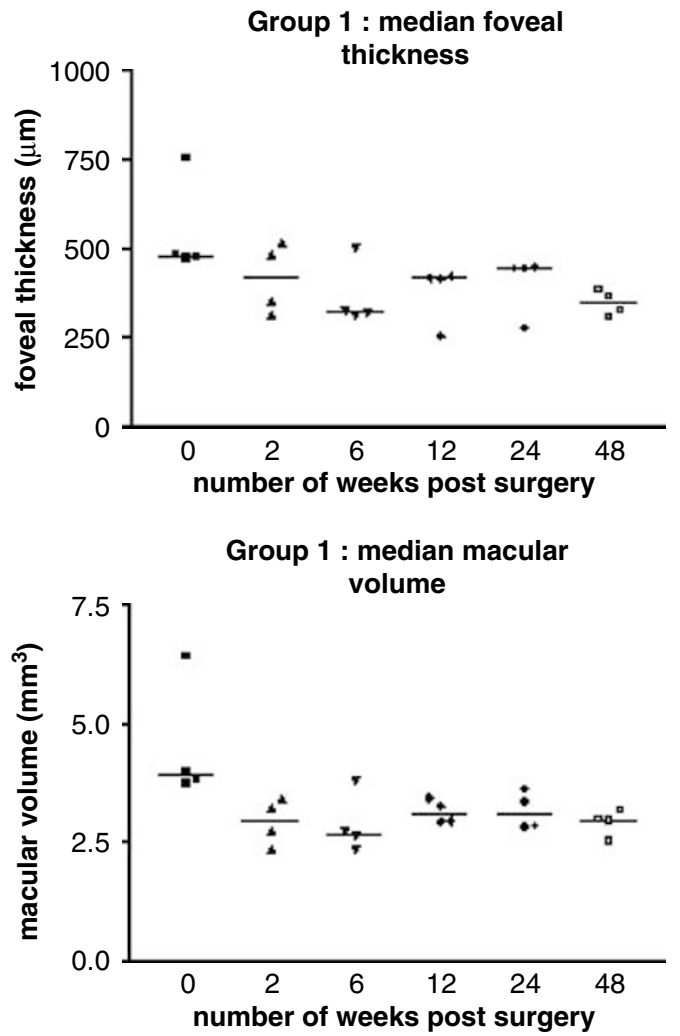

Figure 3 The structural changes in each group after vitrectomy.

lines. This improvement in the ETDRS letters read at 12 months post surgery compared to baseline was significant $(P=0.037)$ (Figure 4$)$. The best corrected median Snellen acuity improved to 6/18 (range $6 / 15$ to $3 / 60$ ).
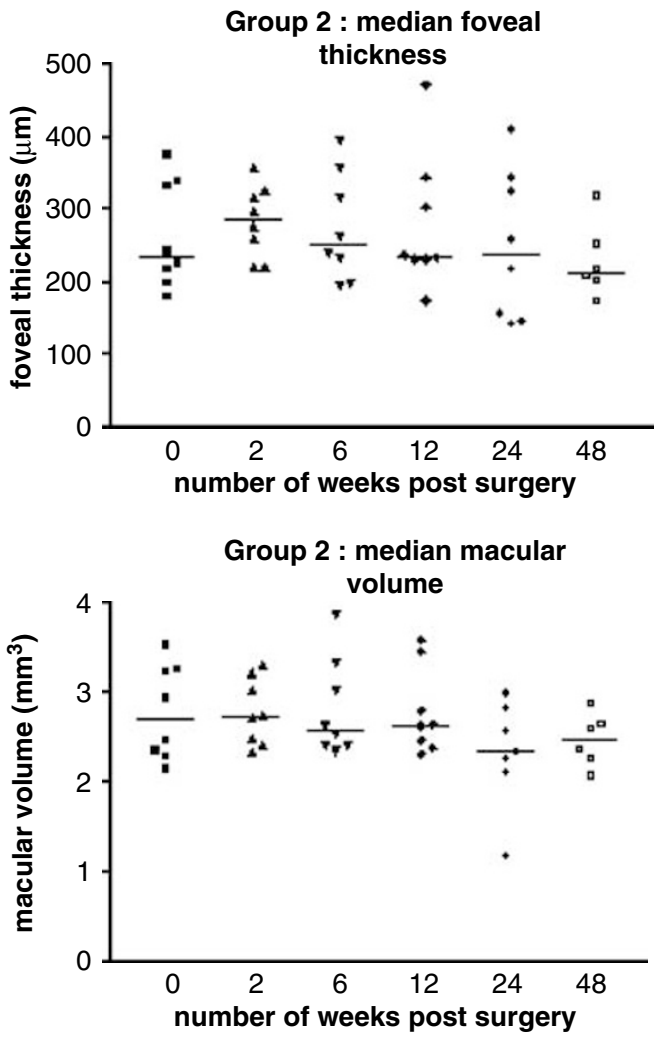

Sub-group analysis

Within each individual macular profile group a small gradual improvement in ETDRS letters read over the 12-month postoperative period was seen: in Group 1 the improvement was 13 letters (range 7-15) and in Group 2 

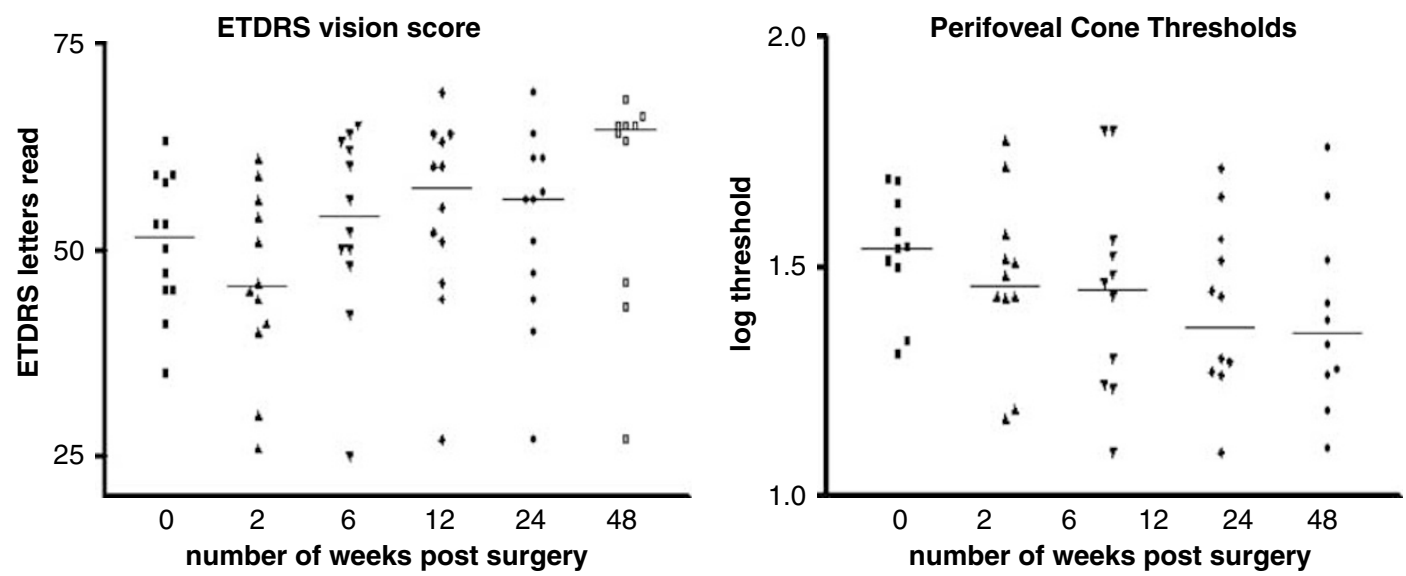

Figure 4 Functional change after vitrectomy for all 12 patients. ETDRS vision and perifoveal cone function gradually improve over the 12 months of follow-up.

it was 14 letters (range 5-11). The corresponding median Snellen acuities at 12 months post PPV were 6/15 (range 6/15-6/45) from a baseline of 6/30 (range 6/18-6/36) for Group 1. For Group 2 they were 6/18 (range 6/15-3/60) compared to the baseline $6 / 30$ (range 6/15-6/60).

\section{FMM (cone threshold) results}

Whole group

Similar to the improved visual acuity, there was a statistically significant improvement in the perifoveal cone thresholds at 12 months compared to baseline for all 12 patients $(P=0.02)$ (Figure 4$)$.

\section{Sub-group analysis}

Although each group showed similar improvement in the cone thresholds these changes did not reach statistical significance $(P>0.05)$, which may be related to the small numbers in each group.

Overlay of the cone thresholds and retinal thickness in the perifoveal $9 \times 9$ degree field, demonstrated three main types of postvitrectomy changes in the perifoveal region at the 12 months post surgery time point. There was a corresponding regional improvement in retinal thickness and cone threshold which occurred in seven eyes. There was also widespread improvement throughout the whole field tested for both thickness and threshold, which was seen in one eye. The final type of change was no improvement in either parameter, which was seen in four eyes.

All three types of change were seen in both anatomical groups described above. Regional improvements were mainly seen in the nasal, temporal, and superior quadrants.

\section{Discussion}

The vitreous is considered to contribute to the pathogenesis of DMO (DME). Hikichi evaluated the association between the natural history of DMO and the state of the vitreous in 82 eyes over a 6-month period and the findings suggested that macular oedema was more likely to resolve in the presence of vitreomacular separation. ${ }^{16}$ Lewis was the first to describe a positive effect of PPV on diabetic patients who had a thickened and taut posterior hyaloid membrane with traction on the macula. There were improvements in both the vision and the degree of macular oedema ${ }^{17}$ and the flattening of the shallow macular detachment which develops secondary to the tractional forces. ${ }^{17-19}$

In this prospective study of 12 eyes of 12 patients with persistent fovea-involving diabetic CSMO, we evaluated the effect of PPV on macular structure and function in a controlled and prospective study and were also able to define two groups of patients with distinct OCT macular profiles. In Group 1, there was a dome-shaped thickening with the posterior vitreous cortex partially detached over the perifoveal area but remaining focally attached at the fovea. In Group 2, there was a diffuse thickening of the macula with no signs of a focal posterior hyaloid separation. For Group 1 the foveal thickness and macular volume was twice and 1.5 times, respectively, compared to the similar indices for Group 2 at baseline. Duguid et $a l^{20}$ were able to describe similar macular profiles in their patients with diabetic CSMO. Giovannini also showed two similar profiles on OCT in 18 diabetic patients with CSMO. ${ }^{21}$ The reason for the two different patterns is not clear but differences in the vitreomacular tractional forces between the two groups may account for the OCT arrangements seen. The dome-shaped profile with its focal attachment of the posterior vitreous cortex on the fovea and adjacent perifoveal detachment could develop from greater tractional forces exerted on the macula and therefore a more thickened fovea and a larger macular volume than the other group with its diffusely thickened macula with no similar arrangement 
of the posterior vitreous cortex. We were unable to tell either on biomicroscopy or on FFA which configuration would be observed on OCT.

Following PPV, there was improvement in the macular profiles and in the structural macular indices for all 12 patients and also for both groups. Collectively there was a $26 \%$ decrease in the foveal thickness and an $18 \%$ decrease in the macular volume for all 12 patients 12 months after surgery. However, for Group 1 the foveal thickness decreased by $33 \%$ and the macular volume by $32 \%$ during the same post surgical period compared to baseline. For Group 2, there was only a 9\% decrease in both these indices for the same time period post surgery. These changes may reflect the differences in the vitreal tractional forces exerted on the macula and may suggest that in those eyes where such forces play a significant contribution to the pathogenesis of diffuse DMO, the removal of these forces by PPV may lead to reductions in retinal thickness especially in the early postoperative period: in Group 1, both these structural indices demonstrated large decreases in the first 2 weeks following surgery compared to Group 2, which showed initial increases for both these parameters.

Following the structural improvement in the macula and fovea, there was also functional improvement in both the visual acuity and perifoveal cone thresholds 12 months after surgery, despite having central CSMO persisting, on average for 15 months (range 12-18 months) prior to surgery and all having been previously treated with macular photocoagulation.

There have been a number of studies evaluating the role of PPV in patients with CSMO and an attached non-thickened hyaloid face..$^{8,9,21-23}$ In all these studies there was a variable level of improvement in visual function. Tachi and Ogino ${ }^{9}$ reported that $53 \%$ of 58 eyes treated improved by 1 line of Snellen acuity. Tachi reported similar levels of improvement in 85 eyes. However, $66-74 \%$ of the eyes undergoing PPV in these two studies also underwent simultaneous phacoemulsification. In a prospective study of 13 eyes for PPV without phacoemulsification by Otani and Kishi, $38 \%$ (5/13) improved by two lines of vision. They also performed OCT assessment of the changes and noted that in those patients where the foveal thickness decreased by more than $50 \%$ the visual acuity improved by two lines. ${ }^{24}$ However, in a recent prospective study by the same authors the 14 eyes of seven patients in which foveal thickness decreased significantly showed no such improvement in the visual acuity. ${ }^{25}$ The authors suggested a longer follow-up is needed to evaluate the efficacy of vitrectomy for DMO.

To date, most studies of vitrectomy for diffuse DMO have been retrospective. Although our study involved a relatively small number of patients, it was prospective, the visual acuity was tested in a standardised format by a masked observer and the follow-up was for a full year after treatment. Also, the differing results for the individual groups defined in this study may also provide an insight into the variable response of resolution of macular oedema and visual function for DMO after PPV.

Relief of the vitreomacular tractional forces and the removal of permeability-inducing cytokines at the vitreomacular interface ${ }^{26}$ may be the likely mechanisms that would allow for decreases in the foveal thickness and improvement in vision post surgery. In a report of inflammatory-induced macular edema, removal of the vitreous containing inflammatory mediators resulted in improvements in visual acuity. ${ }^{27}$ Furthermore, it has recently been suggested that another potential mechanism of action is an increase in oxygenation of the inner retina as a result of PPV. ${ }^{28}$ The increased oxygenation would allow for a decrease in Vascular Endothelial Growth Factor (VEGF) secretion, a potent vasopermeability inducing cytokine. ${ }^{29}$

Previous macular laser treatment may affect the overall degree of visual improvement post surgery. Heij et $a l^{30}$ reported in their retrospective series of 21 eyes that seven eyes without preoperative macular photocoagulation achieved a median percentage improvement of $77 \%$ in vision while 12 eyes with preoperative macular photocoagulation only achieved $15 \%$ improvement in vision, suggesting a significantly better visual outcome in eyes that had undergone no previous macular photocoagulation. In our series, all patients had undergone previous macular laser treatment and the percentage improvement in vision was $20 \%$. Laser photocoagulation can damage foveal cone function. ${ }^{31,32}$ Also in eyes with DMO, there is diminished foveal photoreceptor sensitivity ${ }^{33}$ secondary to abnormal alignment in early macular oedema but with the persistent oedema also secondary to changes in the structure and/or loss of photoreceptors. ${ }^{33}$ Therefore, in CSMO, there are structural and functional changes in the foveal photoreceptors, which affect the level of vision, and in which the destructive effect of macular photocoagulation may worsen. In our patients, the improvement in visual acuity probably reflects changes to the structure and alignment of the photoreceptors resulting from the decrease in the foveal thickness. Larger improvement in the functional indices may not have been possible because of structural changes in the photoreceptor.

In summary, our study showed that PPV can lead to structural and functional improvements in patients with persistent CSMO. We also showed that there are 2 distinct groups of patients with fovea-involving CSMO based on the macular profile as imaged by OCT. There was a $10 \%$ functional improvement and this modest level 
of improvement may relate to the structural and functional changes already present in the photoreceptors secondary to the oedema and prior to macular laser. However a fuller, randomised controlled study is needed to evaluate the true benefit of PPV against standard laser treatment to ascertain the place of PPV in the management of diffuse, persistent macular oedema.

\section{References}

1 Clark J, Grey R, Lim K, Burns-Cox C. Loss of vision before ophthalmic referral in blind and partially sighted diabetics in Bristol. Br J Ophthalmol 1994; 78: 741-744.

2 Klein R, Klein B, Moss S, Davis M, DeMets D. The wisconsin epidemiologic study of diabetic retinopathy. IV. Diabetic macular edema. Ophthalmology 1984; 91: 464-474.

3 Klein R, Klein B, Moss S. Visual impairment in diabetes. Ophthalmology 1984; 91: 1-8.

4 DCCT Research Group. The effect of intensive treatment of diabetes in the development and progression of long-term complications in insulin-dependent diabetes. $N$ Engl J Med 1993; 329: 977-986.

5 UK Prospective Diabetes Study (UKPDS) Group. Intensiveblood-glucose control with sulphonylureas or insulin compared with conventional treatment and risk complications in patients with type 2 diabetes (UKPDS 33). Lancet 1998; 317: 703-713.

6 The Early Treatment Diabetic Retinopathy Study Research Group. Photocoagulation for diabetic macular edema. Early Treatment Diabetic Retinopathy Study Report No 1. Arch Ophthalmol 1985; 103: 1796-1806.

7 Lewis H, Abrams G, Blumenkranz M, Campo R. Vitrectomy for diabetic macular traction edema associated with posterior hyaloidal traction. Ophthalmol 1992; 99: 753-759.

8 Ikeda T, Katano T, Hayashi Y. Vitrectomy for cystoid macular oedema with attached posterior hyaloid membrane in patietns with diabetes. Br J Ophthalmol 1999; 83: 12-14.

9 Tachi N, Ogino N. Vitrectomy for diffuse macular edema in cases of diabetic retinopathy. Am J Ophthalmology 1996; 122: 258-260.

10 Gandofer A, Messmer E, Ulbig M, Kampik A. Resolution of diabetic macular edema after surgical removal of the posterior hyaloid and inner limiting membrane. Retina 2000; 20: $126-133$.

11 Huang D, Swanson E, Lin C, Schuman J, Stinson W, Chang W et al. Optical coherence tomography. Science 1991; 254: 1178-1181.

12 Chaung E, Sharp D, Fitzke F, Kemp C, Holden A, Bird A. Retinal dysfunction in central serous retinopathy. Eye 1987; 1: $120-125$.

13 Chen J, Fitzke F, Pauleikhoff D, Bird A. Functional loss in age-related Bruch's membrane change with choroidal perfusion defect. Invest Ophthalmol Vis Sci 1992; 33: 334-340.

14 Hee M, Puliafito C, Wong C, Duker J, Reichel E, Rutledge B. Quantitative assessment of macular edema with optical coherence topography. Arch Ophthalmol 1995; 113: 1019-1029.

15 Sanchez-Tocino H, Alvarez-Vidal A, Maldonado M, Moreno-Montanes J, Garcia-Layana A. Retinal thickness study with optical coherence tomography in patients with diabetes. Invest Ophthalmol Vis Sci 2002; 43: 1588-1594.
16 Hikichi T, Fuijo N, Akiba J, Azuma Y, Takahashi M, Yoshida A. Association between the short-term natural history of diabetic macular edema and the vitreomacular relationship in type II diabetes mellitus. Ophthalmology 1997; 104: $473-478$.

17 Lewis H, Abrams G, Blumenkranz M, Campo R. Vitrectomy for diabetic macular traction edema associated with posterior hyaloidal traction. Ophthalmology 1992; 99: 753-759.

18 van Efferentere G. Macular oedema caused by contraction of the posterior hylaloid in diabetic retinopathy. J Fr Ophthalmol 1993; 16: 602-610.

19 Harbour J, Smiddy W, Flynn H, Rubsamen P. Vitrectomy for diabetic macular oedema associated with a thickened and taut posterior hyaloid membrane. Am J Ophtalmol 1996; 121: 405-413.

20 Massin P, Duguid G, Erginay A, Haouchine B, Gaudric A. Optical coherence tomography for evaluating diabetic macular edma before and after vitrectomy. Am J Ophthalmol 2003; 135: 169-177.

21 Giovannini A, Amato G, Mariotti C, Scassellati-Sforzolini B. Optical coherence tomography findings in diabetic macular edema before and after vitrectomy. Ophthalmic Surg Lasers 2000; 31: 187-191.

22 Sato Y, Lee Z, Shimada H. Vitrectomy for diabetic cystoid macular edema. Jpn J Ophthalmol 2002; 46: 315-322.

23 Amino K, Tanihara H. Vitrectomy combined with phacoemulsification and intraocular lens implantation for diabetic macular edema. Jpn J Ophthalmol 2002; 46: 455-459.

24 Otani T, Kishi S. Tomographic assessment of surgical outcome of vitreous surgery for diabetic macular edema. Am J Ophthalmol 2000; 129: 487-494.

25 Otani T, Kishi S. A controlled study of vitrectomy for diabetic macular edema. Am J Ophtalmol 2002; 134: 214-219.

26 Kent D, Vinores S, Campochiaro P. Macular oedema: the role of soluble mediators. Br J Ophthalmol 2000; 84: 542-545.

27 Dugel P, Rao N, Ozler S, Liggett P, Smith R. Pars plana vitrectomy for intraocular inflammation-related cystoid macular oedema unresponsive to corticosteroids. Ophthalmology 1992; 99: 1535-1541.

28 Stefansson H-L. The therapeutic effects of retinal laser treatment and vitrectomy. A theory based on oxygen and vascular physiology. Acta Ophthalmol Scand 2001; 79 435-440.

29 Senger D, Galli S, Dvorak A, Perruzzi C, Harvey V, Dvorak $\mathrm{H}$. Tumour cells secrete a vascular permeability factor that promotes of ascites fluid. Science 1983; 219: 983-985.

30 Heij E, Hendrikse F, Kessels A, Derhaag P. Vitrectomy results in diabetic macular oedema without evident vitreomacular traction. Graefe's Arch Clin Exp Ophthalmol 2001; 239: 264-270.

31 Weiner A, Christopoulos V, Gussler C, Adams D, Kaufman $\mathrm{S}, \mathrm{Kohn} \mathrm{H}$ et al. Foveal cone function in nonproliferative diabetic retinopathy and macular edema. Invest Ophthalmol Vis Sci 1997; 38: 1443-1449.

32 Greenstein V, Holopigian K, Hood D, Seiple W, Carr R. The nature and extent of retinal dysfunction associated with diabetic macular edema. Invest Ophthalmol Vis Sci 2000; 41: 3643-3654.

33 Lardenoye C, Probst K, DeLint P, Rothova A. Photoreceptor function in eyes with macular edema. Invest Ophthalmol Vis Sci 2000; 41: 4048-4053. 